Journal of Mathematics and Statistics Studies

ISSN: 2709-4200

DOI: 10.32996/jmss

Journal Homepage: www.al-kindipublisher.com/index.php/jmss

\title{
On Mixture GARCH Models: Long, Short Memory and Application in Finance
}

\author{
Madjda Amrani' 8(D) and Halim Zeghdoudi ${ }^{2}$ (D) \\ ${ }^{12}$ LaPS laboratory, Faculty of Sciences, University of Badji-Mokhtar University, BP12 Annaba 23000-Algeria \\ $\triangle$ Corresponding Author: Halim Zeghdoudi, E-mail: halimzeghdoudi77@gmail.com
}

\section{ARTICLE INFORMATION}

Received: April 08, 2021

Accepted: June 14, 2021

Volume: 2

Issue: 2

DOI: $10.32996 /$ jmss.2021.2.2.1

\section{KEYWORDS}

GARCH; IGARCH; EGARCH; PARCH;

MMGRACH; long and short

memory; Mixture Models, MSC

2010 No.:62P05, 62H12, 91B70

\section{ABSTRACT}

In this work, we study the famous model of volatility; called model of conditional heteroscedastic autoregressive with mixed memory MMGARCH for modeling nonlinear time series. The MMGARCH model has two mixing components, one is a GARCH short memory and the other is GARCH long memory. the main objective of this search for finds the best model between mixtures of the models we made (long memory with long memory, short memory with short memory and short memory with long memory) Also, the existence of its stationary solution is discussed. The Monte Carlo experiments demonstrate we discovered theoretical. In addition, the empirical application of the MMGARCH model $(1,1)$ to the daily index DOW and NASDAQ illustrates its capabilities; we find that for the mixture between APARCH and EGARCH is superior to any other model tested because it produces the smallest errors.

\section{Introduction}

In financial literature, the risk or the volatility of an asset return is specified as the conditional variance empirically. Volatility modeling is the generalized autoregressive conditional heteroscedasticity (GARCH) processes introduced by Engle (1982) and Bollerslev (1986). Autoregressive Conditional Heteroskedasticity (ARCH) models are specifically designed to model and forecast conditional variances. The variance of the dependent variable is modeled as a function of past values of the dependent variable and independent or exogenous variables.

ARCH models were introduced by Engle (1982) and generalized as GARCH (Generalized ARCH) by Bollerslev (1986) and Taylor (1986). These models are widely used in various branches of econometrics, especially in financial time series analysis. See Bollerslev, Chou, and Kroner (1992) and Bollerslev, Engle, and Nelson (1994) for surveys.

Li et al. (2013) proposed the Mixture Memory GARCH. The MMGARCH combines GARCH processes with short memory (GARCHpart) and long memory(FIGARCH-part) in volatility and provides a time-dependent and stochastic decision on whether to use short or long memory in each modeling step. Tony Klein and Thomas Wather are the first to test the MMGARCH on oil price returns and find it to be superior to non-switching/non-maxing GARCH models.

The article is organized as follows. Section 2 is devoted to present the different models GARCH and introduces the MM-GARCH mixture memory GARCH. Finally, an application study of daily DOW and NASDAQ data from 1 January 1995 to 24 December 2018 with 8640 observations is given.

\section{Methodology}

2.1 ARCH Model: Consider the first-order autoregressive conditional heteroskedasticity $(\mathrm{ARCH})$ process, which is given by:

$$
u_{t}=\varepsilon_{t} \sqrt{h_{t}} \varepsilon_{t} \square N(0,1)
$$

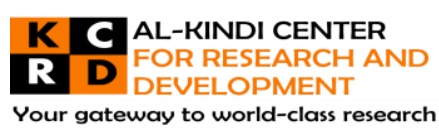

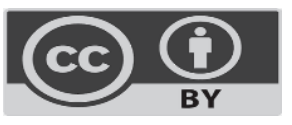

Published by Al-Kindi Center for Research and Development. Copyright (c) the author(s). This open access article is distributed under a Creative Commons Attribution (CC-BY) 4.0 license 


$$
y_{t}=\mu_{t}+u_{t} h_{t}=\alpha_{0}+\sum_{i=1}^{p} \alpha_{i} u_{t-i}^{2}
$$

It has to hold that $\alpha_{0}, \alpha_{i} \geq 0$ for all $i=1, \ldots, \mathrm{p}$ and $\sum_{i=1}^{p} \alpha_{i}<0$ where $u_{t}$ is the return and is assumed here to be an ARCH (p) process. $\varepsilon_{t}$ is a white noise with zero mean and variance of one. $\varepsilon_{t}$ May or may not follow a normal distribution.

2.2 GARCH Model: This model can now be extended to a $\operatorname{GARCH}(p, q)$, where $p$ is the number of lags of the conditional variance and $q$ is the number of lags of the squared error. The GARCH $(p, q)$ process can be described as follows:

$$
\begin{aligned}
& h_{t}=\alpha_{0}+\sum_{i=1}^{p} \alpha_{1} u_{t-i}^{2}+\sum_{j=1}^{q} \beta_{j} h_{t-j}^{2} \\
& \sum_{i=1}^{p} \alpha_{i}+\sum_{i=1}^{q} \beta_{i}<0
\end{aligned}
$$

2.3 APARCH Model: The proliferation of GARCH models has inspired some authors to define families of GARCH models that would accommodate as many individuals as models as possible. The Asymmetric Power ARCH (Ding, Engle and Granger, 1993), the $\operatorname{APARCH}(p, q)$ model can be expressed as:

$$
h_{t}^{\delta}=\omega+\sum_{i=1}^{p} \alpha_{i}\left(\left|u_{t-i}\right|-\gamma_{i} u_{t-i}\right)^{\delta}+\sum_{j=1}^{q} \beta_{j} h_{t-j}^{\frac{\delta}{2}}
$$

The APARCH equation is supposed to satisfy the following conditions: $\omega>0, \delta \geq 0, \alpha_{i} \geq 0,-1<\gamma_{i}<1, i=1, \ldots, p \beta_{j} \geq 0$ $j=1, \ldots, \mathrm{q}$.

2.4 IGARCH Model: This model is suggested by Engle \& Bollerslev (1986) firstly to capture the long memory effect of the volatility process. IGARCH $(p, q)$ is the standard $\operatorname{GARCH}(p, q)$ but with

$$
\begin{aligned}
& \sum_{i=1}^{p} \alpha_{i}+\sum_{i=1}^{q} \beta_{i}=1 \\
& h_{t}=\alpha_{0}+\sum_{i=1}^{p} \alpha_{i} u_{t-i}+\sum_{i=1}^{q} \beta_{i} h_{t-i}
\end{aligned}
$$

2.5 EGARCH Model: Nelson (1991) presents a model that is known as the Exponential GARCH (EGARCH). This model has many advantages in comparison to the original GARCH model. The EGARCH $(p, q)$ model is given by

The function $\mathbf{g}\left(\varepsilon_{t-i}\right)$ is piecewise linear in $\varepsilon_{t}$

$$
g\left(\varepsilon_{t-i}\right)=a_{i} \varepsilon_{t-i}+b_{i}\left(\left|\varepsilon_{t-i}\right|-E\left|\varepsilon_{t-i}\right|\right)
$$

\subsection{Mixture Memory GARCH Model}

Motivated by Li and al. (2013), we incorporate a GARCH $(1,1)$ and FIGARCH $(1, d, 0)$ component for variance modeling. The model is applied by Klein and Walther(2016) on oil prices with $\operatorname{GARCH}(1,1)$ and $\operatorname{FIGARCH}(1, \mathrm{~d}, 1)$ Let $\varepsilon_{t} \square N(0.1)$ i.i.d.for all $\mathrm{t}=1, \ldots, \mathrm{n}$. A random mixture between the GARCH and the FIGARCH component is incorporated by introducing a sequence of Bernoulli random variables $\left(Z_{t}\right)_{t=1, \ldots, n}$, where $\mathrm{P}\left[Z_{t}=1\right]=\alpha_{t}$ the mixture proportion $\alpha_{t} \in[0.1]$ is addressed at a later point [10]. The Mixture Memory GARCH (MMGARCH) is then defined as: 
$y_{t}=\mu_{t}+u_{t}$

$u_{t}=\varepsilon_{t} \sqrt{h_{t}}$

$h_{t}=\alpha_{t} h_{1, t}+\left(1-\alpha_{t}\right) h_{2, t}$

For $\mathrm{t}=1, \ldots . \mathrm{n}$, when $h_{1, t}$ denotes the GARCH models.

2.7 New Mixture Memory GARCH Model

$$
\begin{aligned}
& h_{t}=\sum_{i=1}^{n} \alpha_{i} h_{\mathrm{i}, t} \\
& \sum_{i=1}^{n} \alpha_{i}=1
\end{aligned}
$$

\subsection{Quality Evaluation of Forecasts}

The forecast error statistics used in this study are the root mean square error (RMSE), mean absolute error (MAE) and the mean absolute percentage error (MAPE). These forecast error statistics are defined by:

\begin{tabular}{|l|l|l|}
\hline Mean Square Error (MSE) & Root Mean Square Error (RMSE) & Mean Absolute Error (MAE) \\
\hline MSE $=\frac{1}{N} \sum_{t=1}^{N}\left(\widehat{h_{t}}-h_{t}\right)^{2}$ & RMSE $=\sqrt{\frac{1}{N} \sum_{t=1}^{N}\left(\widehat{h_{t}}-h_{t}\right)^{2}}$ & MAE $=\frac{\mathbf{1}}{N} \sum_{t=1}^{N}\left[\widehat{h_{t}}-h_{t}\right]$ \\
\hline
\end{tabular}

Where $\mathrm{N}$ denote the number of observation, $\widehat{\mathrm{h}}_{\mathrm{t}}$ denotes the forecasted variance at time $\mathrm{t}$ and $\mathrm{h}_{\mathrm{t}}$ denotes the actual, realized variance.

\section{APPLICATION TO EXCHANGE RATES}

This section gives an application and simulation using the daily DOW and NASDAQ data for comparing the different models.

\subsection{REAL DATA ANALYSIS}

We consider the daily DOW and NASDAQ data from 1January 1995 to 24 December 2018 with 8640 observations in this application.

We make an overview descriptive statistics of the return of indices DOW and NASDAQ:

\begin{tabular}{|l|l|l|}
\hline & \multicolumn{1}{|c|}{ DOW } & NASDAQ \\
\hline Observation & 8640 & 8546 \\
\hline Mean & 11771.18 & 2887.296 \\
\hline Median & 10725.43 & 2346.9 \\
\hline VAR & 24294966 & 2618904 \\
\hline Standard deviation & 4928.992 & 1618.303 \\
\hline Max & 3593.35 & 743.58 \\
\hline Min & 26828.39 & 8109.69 \\
\hline Skewness & 0.872947 & 1.269671 \\
\hline Kurtosis & 0.7055925 & 0.9893846 \\
\hline
\end{tabular}

Table 1.Descriptive statistics for DOW and NASDAQ returns 01/01/1995 -24/12/2018 


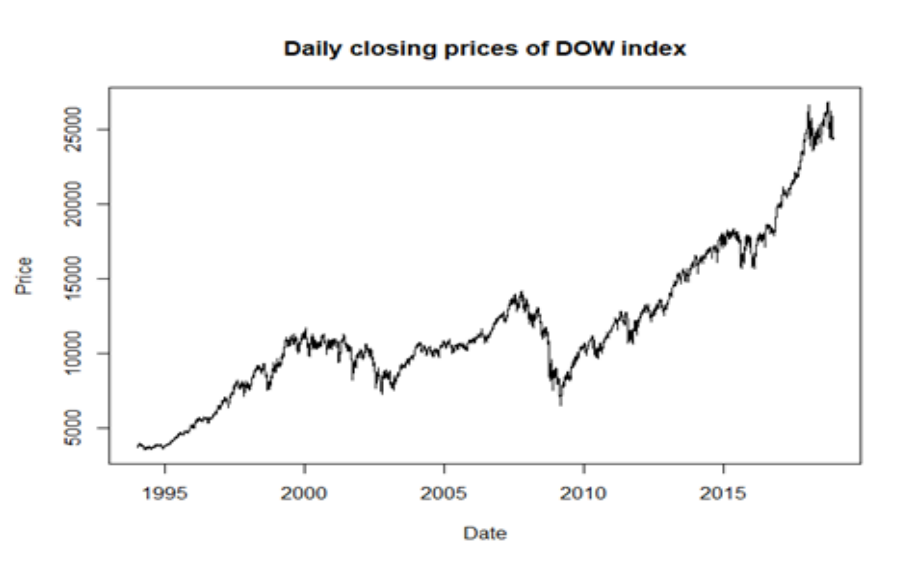

Figure 1.NASDAQ returns 01/01/1995-24/12/2018 $n=8546$

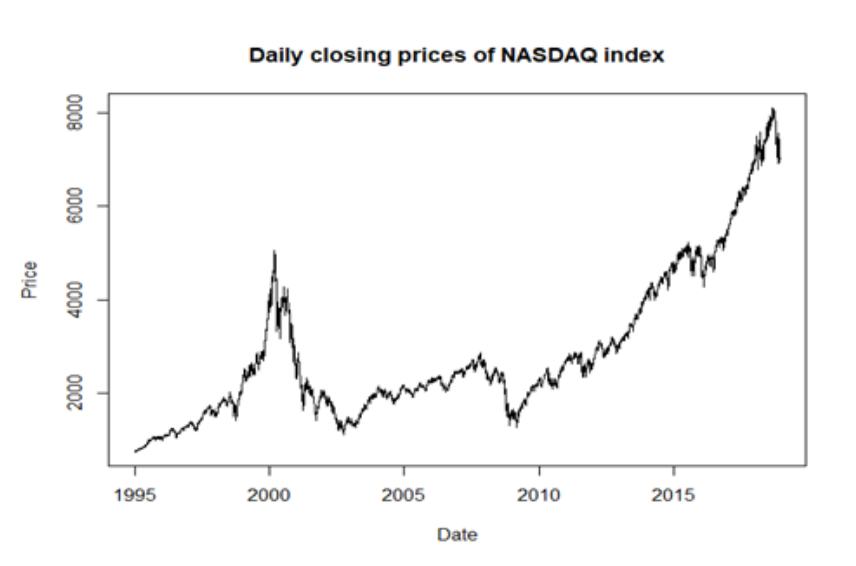

Figure 2.DOW returns 01/01/1995-24/12/2018 $n=8640$

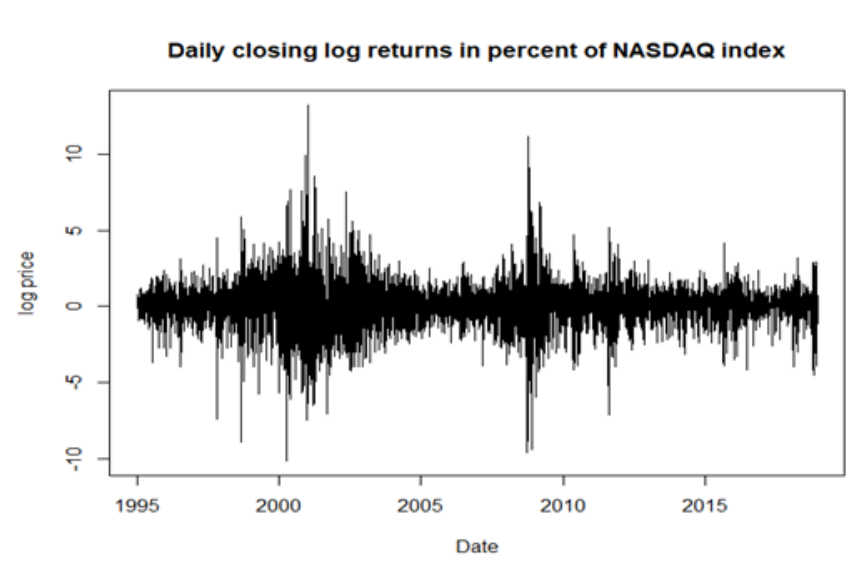

Figure 3.NASDAQlog returns 01/01/1995-24/12/2018 $n=8546$

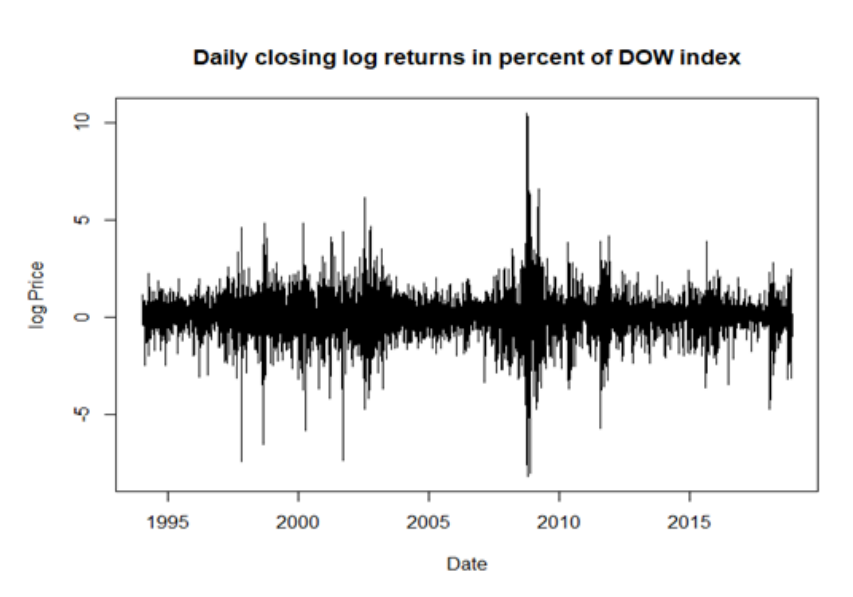

Figure 4.DOW log returns 01/01/1995-24/12/2018 n=8546

In this section, we apply the proposed HGARCH model as well as $\operatorname{GARCH}(1,1), \operatorname{IGARCH}(1,1), \operatorname{EGARCH}(1,1), \operatorname{APARCH}(1,1)$ and FIGARCH $(1,1,1)$ models the volatilities of two financial time series: daily prices of DOW indices and: daily prices of DOW indices

\begin{tabular}{|l|l|l|l|l|l|}
\hline & GARCH $(1,1)$ & IGARCH $(1,1)$ & EGARCH $(1,1)$ & APARCH $(1,1)$ & FIGARCH $(1,1,1)$ \\
\hline$\mu$ & 0.064501 & 0.065157 & 0.033717 & 0.029016 & 0.064501 \\
\hline$\omega$ & 0.017092 & 0.12447 & -0.001686 & 0.025873 & 0.017082 \\
\hline$\alpha$ & 0.106616 & 0.117659 & -0.124606 & 0.086446 & 0.986337 \\
\hline$\gamma$ & 0.879727 & 0.882341 & 0.972887 & 0.905236 & 0.879783 \\
\hline$\delta$ & - & - & - & 1.040182 & 0.000100 \\
\hline Log-likelihood & -8220.873 & -8228.02 & -8107.166 & -8088.178 & -8220.878 \\
\hline BIC & 2.6237 & 2.6246 & 2.5889 & 2.5842 & 2.6237 \\
\hline
\end{tabular}

Table 2. Parameter estimates of DOW log return 01/01/1995-24/12/2018 n=8546 


\begin{tabular}{|l|l|l|l|l|l|}
\hline & GARCH $(1,1)$ & $\operatorname{IGARCH}(1,1)$ & $\operatorname{EGARCH}(1,1)$ & APARCH $(1,1)$ & FIGARCH $(1,1,1)$ \\
\hline$\mu$ & 0.084972 & 0.084157 & 0.043439 & 0.044063 & 0.085097 \\
\hline$\omega$ & 0.024317 & 0.018166 & 0.012401 & 0.027394 & 0.023642 \\
\hline$\alpha$ & 0.100626 & 0.109084 & -0.094470 & 0.089322 & 0.988988 \\
\hline$\beta$ & 0.888589 & 0.890616 & 0.979260 & 0.906713 & 0.891224 \\
\hline$\delta$ & - & - & & 0.564797 & 0.005000 \\
\hline log-likelihood & -9727.834 & -9733.389 & -9658.785 & -9643.232 & -9728.182 \\
\hline BIC & 3.2333 & 3.2337 & 3.2119 & 3.2081 & 3.2334 \\
\hline
\end{tabular}

Table 3. Parameter estimates of NASDAQ log returns 01/01/1995-24/12/2018 $n=8546$

\section{Conclusion}

This work studied MMGARCH model, with its two mixed components. One GARCH is parked, the other is GARCH to long memory or a short memory. An application study of daily DOW and NASDAQ data from 1 January 1995 to 24 December 2018 with 8640 observations is given. We took the different combinations mentioned above that brought out the new models. In conclusion, we have judged that the 4 th model is the best. We compare the results of the variance predictions obtained and the calculated errors are listed in the following tables (out-of-sample forecast for DOW log returns and out-of-sample forecast for NASDAQ log returns With regard to RMSE and MAE, We find that both APARCH and EGARCH blends are superior to any other model tested because it produces the smallest errors.

\section{References}

[1] Charles, A., \& Darné, O. (2019). The accuracy of asymmetric GARCH model estimation. International Economics, 157, $179-202$.

[2] Cheng, X., Yu, P. L., \& Li, W. K. (2009). On a dynamic mixture GARCH model. Journal of Forecasting, 28(3), 247-265.

[3] Engle, R. F. (1982). Autoregressive conditional heteroscedasticity with estimates of the variance of United Kingdom inflation. Econometrica: Journal of the econometric society, 987-1007.

[4] Hamdi, F., \& Souam, S. (2013, April). Mixture periodic GARCH models: Applications to exchange rate modeling. In 2013 5th International Conference on Modeling, Simulation and Applied Optimization (ICMSAO) (pp. 1-6). IEEE.

[5] Klein, T., \& Walther, T. (2016). Oil price volatility forecast with mixture memory GARCH. Energy Economics, 58, 46-58.

[6] Li, M., Li, W. K., \& Li, G. (2013). On mixture memory GARCH models. Journal of Time Series Analysis, 34(6), 606-624.

[7] Meitz, M., Preve, D., \& Saikkonen, P. (2021). A mixture autoregressive model based on Student'st-distribution. Communications in Statistics-Theory and Methods, 1-76.

[8] Wong, C. S., \& Li, W. K. (2000). On a mixture autoregressive model. Journal of the Royal Statistical Society: Series B (Statistical Methodology), 62(1), 95-115.

[9] Wong, C. S., \& Li, W. K. (2001). On a logistic mixture autoregressive model. Biometrika, 88(3), 833-846.

[10] Wong, C. S., \& Li, W. K. (2001). On a mixture autoregressive conditional heteroscedastic model. Journal of the American Statistical Association, 96(455), 982-995.

[11] Zhang, Z., Li, W. K., \& Yuen, K. C. (2006). On a mixture GARCH time-series model. Journal of Time Series Analysis, 27(4), $577-597$. 